\title{
Bacteria versus parasites: Effect of Lactobacillus rhamnosus cell- free supernatant on the sporulation of coccidian oocysts in vitro and in vivo
}

\author{
Abdul-Lateef Molan [Professor of Medical Parasitology] \\ Department of Biology, College of Sciences, Diyala University, Diyala, Iraq
}

\begin{abstract}
The purpose of this study was to determine if the probiotic bacterium, Lactobacillus rhamnosus, generates compounds that affect the sporulation of the oocysts of three species of Eimeria using an in vitro assay. In addition, anticoccidial effects of L. rhamnosus cell-free supernatant (CFS)-based diets were evaluated in chickens following experimental infection with Eimeria tenella and the anticoccidial effect was assessed using fecal oocyst shedding and the sporulation process. L. rhamnosus was grown in Mann-Rogosa-Sharpe (MRS) broth for 48 h at $37^{\circ} \mathrm{C}$ anaerobically and the cells were removed from the broth by centrifugation to get the CFS. Exposure of the oocysts of the 3 species of Eimeria, namely E. acervulina, E. tenella and E. maxima, to 4-fold diluted CFS resulted in $37 \%, 33 \%$ and $46 \%$ inhibition of sporulation, respectively while exposure to undiluted CFS resulted in 100\% inhibition of sporulation in all species. Chickens fed $1 \%$ or $3 \%$ CFSsupplemented diets in E. tenella-infected groups shed significantly less $(P<0.0001)$ oocysts than their counterparts fed the control diet. More importantly, the sporulation rate of the oocysts recovered from the CFSsupplemented birds was significantly lower than that of the oocysts recovered from the infected birds fed the control diet $(P<0.0001)$. The results showed for the first time that CFS from L. rhamnosus cultures has anticoccidial activity.
\end{abstract}

Keywords: Coccidiosis, oocysts, sporulation, Lactobacillus rhamnosus, cell-free supernatant, anticoccidial activity

\section{Introduction}

The coccidia are a group of protozoan parasites that are responsible for serious diseases in both animals and humans. Coccidiosis, caused by infection with Eimeria species affects cattle, deer, sheep, goats, pigs, horses, rabbits, turkeys, ducks and poultry [1]. Coccidiosis is considered to be one of the most economically important diseases of domestic poultry throughout the world [2].

Although the use of anticoccidial drugs has provided the basis for the rapid growth of intensive poultry production and the increased availability of high quality poultry products to the consumers, the increasing resistance of avian coccidia to currently used anticoccidial drugs [3-4], coupled with the increasing costs of developing new drugs and the public's distrust of drug-treated meat demonstrate the urgent need to explore alternative methods of controlling this disease.

Probiotics are viable, non-pathogenic microorganisms that, when ingested, benefit the health of the host by balancing the intestinal microbiota via inhibiting the growth of undesirable bacteria and by fermenting the indigestible substrates that reach the lower part of the gastrointestinal tract. Many studies report on the inhibition of a wide range of pathogenic micro-organisms by lactic acid bacteria under in vitro and in vivo conditions, such as Escherichia coli, Salmonella, and rotavirus [5-7]. Mice fed Lactobacillus acidophilus (LA) or Lactobacillus reuteri (LR) and experimentally infected with bovine Cryptosporidium parvum shed lower numbers of oocysts and had a shortened duration of shedding compared to non-Lactobacillus fed controls [8-9]. New-born piglets naturally infected with $C$. parvum and given L. acidophilus and Bifidobacterium species, intragastrically had less severe diarrhea, dehydration and histological lesions compared to litter mates not receiving LA [10].

Foster et al. [11] assessed in vitro the effects of cell free bacterial supernatants of LA and LR on bovine $C$. parvum oocyst viability and found that the bacterial culture supernatants reduced $C$. parvum oocyst viability by up to $81 \%$, suggesting that the mechanism seen in vitro may be production of an anti-microbial compound or a variety of compounds.

This study is a part of a programme aiming to find alternative strategies to treat coccidiosis using natural products. The purpose of this study was to determine if the probiotic bacterium, L. rhamnosus, generates compounds that affect the sporulation of the oocysts of three species of Eimeria, namely E. acervulina, E. tenella and E. maxima under laboratory conditions. In addition, anticoccidial effects of $L$. rhamnosus cell-free supernatant (CFS)-based diets in chickens following experimental infection with E. tenella were evaluated. 


\subsection{Bacterial strain and supernatant preparation}

\section{Materials and Methods}

Lacobacillus rhamnosus strain NZRM 299 was used in this study. This strain was obtained from the Environmental Science and Research (ESR), New Zealand. Lacobacillus rhamnosus was grown overnight in Mann-Rogosa-Sharpe (MRS) broth at $37{ }^{\circ} \mathrm{C}$ anaerobically (85\% nitrogen, $10 \%$ hydrogen, 5\% carbon dioxide). Cultures were sub-cultured once and inoculated in $10 \mathrm{ml}$ of MRS broth. After reaching stationary phase $(24 \mathrm{~h})$, cells were removed from broth by centrifugation at $6500 \mathrm{~g}$ at $4{ }^{\circ} \mathrm{C}$ for 10 minutes. Supernatants were use for both in vitro and in vivo experiments. Sporulation inhibition bioassay was used to evaluate the anticocccidial activity of cell-free supernatant (CFS).

\subsection{Experimental design and sporulation inhibition assay}

The fecal samples collected from chickens infected experimentally with pure oocysts of each species of Eimeria were obtained from Avivet Ltd, Palmerston North, New Zealand (www.avivet.co.nz). The samples were brought to the laboratory in a $50-\mathrm{ml}$ centrifuge tubes containing $2 \%(\mathrm{w} / \mathrm{v})$ potassium dichromate to prevent bacterial degradation of oocysts during transportation.

The samples were processed as described by Molan et al. [12]. Briefly, a 2-g fecal sample was transferred from the centrifuge tube into a glass beaker and mixed with $1 \mathrm{ml}$ of $2 \%(\mathrm{w} / \mathrm{v})$ potassium dichromate, before adding $41 \mathrm{ml}$ of water. The mixture was vortexed for $1 \mathrm{~min}$ and the resulting suspension was strained through double-layered cheesecloth into a beaker. The filtrate was thoroughly mixed before transferring $5 \mathrm{ml}$ to a centrifuge tube and then centrifuged for $5 \mathrm{~min}$ at $1000 \times \mathrm{g}$. After removing the supernatant, the pellet was resuspended in $5 \mathrm{ml}$ of saturated salt solution. After vortexing for $1 \mathrm{~min}$, a sub-sample was transferred into a McMaster chamber, using a disposable plastic pipette. The tube was vortexed again before the second chamber of the McMaster slide was filled with a second sub-sample. The preparation was left for 5 min before counting, allowing the oocysts to float up. All the oocysts under the grid of each chamber in the McMaster slide were counted using $10 \times$ objectives and the mean of the two counts was calculated. This process was repeated twice and a count of one oocyst was equivalent to 300 (45 ml-the total volume of the original suspension of feces/0.15 ml-the volume of the sample examined) oocysts/sample [13]. The number of unsporulated oocysts in the original aqueous suspension of faeces was adjusted to one million oocysts per ml.

The unsporulated oocysts were used within 2-4 hours of arrival to the laboratory. In this bioassay, the unsporulated oocysts of each strain of Eimeria were exposed to 6 concentrations (undiluted and diluted;1-32 folds in water) of the cell-free supernatant (CFS) for 48-72 h at 25-29 ${ }^{\circ} \mathrm{C}$ depending on the species of Eimeria using water bath to maintain constant temperature. The oocysts were gently aerated with an air pump away from sun light.

At the end of the incubation, the sporulated oocysts were washed twice in tap water and stored at $4{ }^{\circ} \mathrm{C}$ until being counted. The number of sporulated and non-sporulated oocysts was counted and the percent sporulation was estimated by counting the number of sporulated oocysts in a total of 200 oocysts. The oocysts were slightly flattened under the pressure of a cover slip to better illustrate morphology. Oocysts and sporocysts were viewed using phase contrast microscopy. In addition, the numbers of sporocysts within each sporulated oocyst were counted and the oocysts with 4 sporocysts were considered sporulated regardless the shape and size of the sporocysts.

\subsection{Eimeria tenella infection and assessment of fecal oocyst production and sporulation rate}

The sporulated oocysts of E. tenella, obtained from Avivet Ltd, Palmerston North, New Zealand. Chickens were orally infected with 10,000 sporulated oocysts of E. tenella at 2 weeks of age and transferred to wire-floored grower cages (five birds/ cage).

Oocyst production and shedding were assessed as described previously [12]. Briefly, fecal droppings from each cage ( 3 cages/treatment) were collected for $24 \mathrm{~h}$, starting at 9.0 am on day 6 post-infection until 9.0 am on day 7 post-infection, and the oocysts were counted microscopically using a McMaster counting chamber. The total number of oocysts was calculated using the formula: total oocysts/bird = oocyst count $\mathbf{x}$ dilution factor $\mathrm{x}$ (fecal sample volume/counting chamber volume)/number of birds per cage.

\subsection{Experimental scheme}

Male broiler chicks (45, Ross strain), one day old weighting 35 to $45 \mathrm{~g}$ were kept in battery cages of varying sizes, depending on body size, and fed $a d$ lib ration and water. Starter and grower rations representative of local formulations (65\% wheat, $25 \%$ soyameal, $6 \%$ meat and bone meal, $2 \%$ vegetable oil (w/w)) were fed in mashed form. Chicks, which died during the first $48 \mathrm{~h}$ of the study, were replaced with a healthy chicken of similar body weight. Experimental procedures were approved by the Massey University Animal Ethics Committee and complied with the Animal Welfare Act(s) and Regulations of New Zealand. 


\subsection{Housing and management}

Upon arrival, day-old broiler chicks were assigned to pens ( 5 birds/ pen) in 3-tier electrically heated battery brooders $(60 \times 60 \mathrm{~cm})$ in an environmentally controlled room with 24-h fluorescent lighting. The chickens were observed at least four times daily for signs of ill-health throughout the course of the study and a daily log kept of all activities relating to the chickens. Dead birds were removed immediately. Each of the three treatments was then randomly assigned to 3 pens of five chicks each. During day 1 to 12 , all birds were fed the basal ration (without any added coccidiostat). The birds were transferred to colony cages $(48 \times 75 \mathrm{~cm})$ in an environmentally controlled room on day 12 . The cell-free supernatants were added to milled diet ingredients before pelleting. The treatment diets were introduced on day 12 ( 2 days before $E$. tenella challenge) until day 21. Room temperature was maintained at $32 \pm 1{ }^{\circ} \mathrm{C}$ during the first week and gradually decreased to $24{ }^{\circ} \mathrm{C}$ by the end of the third week. Feed and water were provided ad libitum.

This experiment compared the efficacy of one control group (control diet without CFS) with two groups fed CFS (diet supplemented with $1 \%$ and 3\% CFS, respectively). On day 14, all birds were challenged with 10,000 sporulated oocysts of E. tenella via oral gavage. Accordingly, birds in the treated groups received CFS-supplemented feed for 10 days (from day 12 to 21). On day 21, all control and experimental birds were humanely euthanased and both caeca from each bird were removed and one gram of the contents from each cecum was weighted and put into a vial containing $2 \mathrm{ml}$ of tap water, vortexed and incubated in a digital water bath at $29{ }^{\circ} \mathrm{C}$ for 3 days. At the end of the incubation, the numbers of sporulated and non-sporulated oocysts were counted and the \% sporulation was determined as mentioned above.

\subsection{Statistical analysis}

Data are presented as the mean \pm S.E.M. A One-way ANOVA, followed by Tukey's test, was used to detect significance among groups. $\mathrm{P}<0.05$ was considered to be statistically significant.

\section{Results}

3.1. Effect of Lactobacillus rhamnosus cell-free supernatant on sproulation of Eimeria tenella, E. acervulina and E. maxima oocysts in vitro

A series of experiments have been undertaken to determine if the probiotic bacterium, L. rhamnosus, generates compounds that affect the sporulation of the oocysts of three species of Eimeria under the labrotory conditions and the results are shown in Fig. 1. In control incubations (without CFS) containing oocysts of $E$. acervulina, $85 \%$ of the oocysts sporulated (Fig. 1A). In incubations containing either 16-fold or 32-fold diluted CFS, no difference in the \% sporulation in comparison with the oocysts in the control incubation. Exposure of the oocysts to 1,2, 4 and 8-fold diluted CFS resulted in 76\%,54\%,37\% and 12\% inhibition of sporulation, respectively while exposure to undiluted CFS resulted in 100\% inhibition of sporulation (Fig. 1B).

In control incubations (without CFS) containing oocysts of E. tenella, $89 \%$ of the oocysts were managed to sporulate (Fig. 1A) while exposure to undiluted CFS resulted in 100\% inhibition of sporulation (Fig. 1B). Exposure the oocysts to CFS diluted 1, 2, 4, 8, 16 and 32 folds resulted in 73\%, 61\%, 33\%, 15\%, 3\% and $2 \%$ inhibition of sporulation, respectively relative to the control incubations without CFS (Fig. 1B).

It can be seen from Fig. 1A that in control incubation, $86 \%$ of the oocysts of E. maxima reached full sporulation whereas in incubations containing 16 -fold and 32 -fold diluted CFS, only $1 \%$ and $4 \%$ of the oocysts, respectively were able to sporulate ( $\mathrm{P}>0.05$ relative to control incubation). Exposure of the oocysts to CFS diluted 2, 4 and 8 folds resulted in 73\%, 46.2\% and 41\% inhibition of sporulation ( $\mathrm{P}<0.0001$ compared to control incubations), respectively while exposure to undiluted or 1-fold diluted CFS resulted in 100\% inhibition of sporulation when compared to the oocysts in the control incubation (Fig. 1B). No abnormal sporocysts were observed inside the oocysts recovered from either control incubations or those recovered from incubations containing CFS.

The $E$. maxima oocysts seemed to be more susceptible to the harmful effect of CFS than those of $E$. tenella and E. acervulina. For example, exposure of the oocysts of E. tenell, E. acervulina and E. maxima to 1fold diluted CFS resulted in inhibition of the sporulation by $76 \%, 73 \%$ and $100 \%$, respectively when compared to the oocysts in the control incubation (Fig. 1B). 

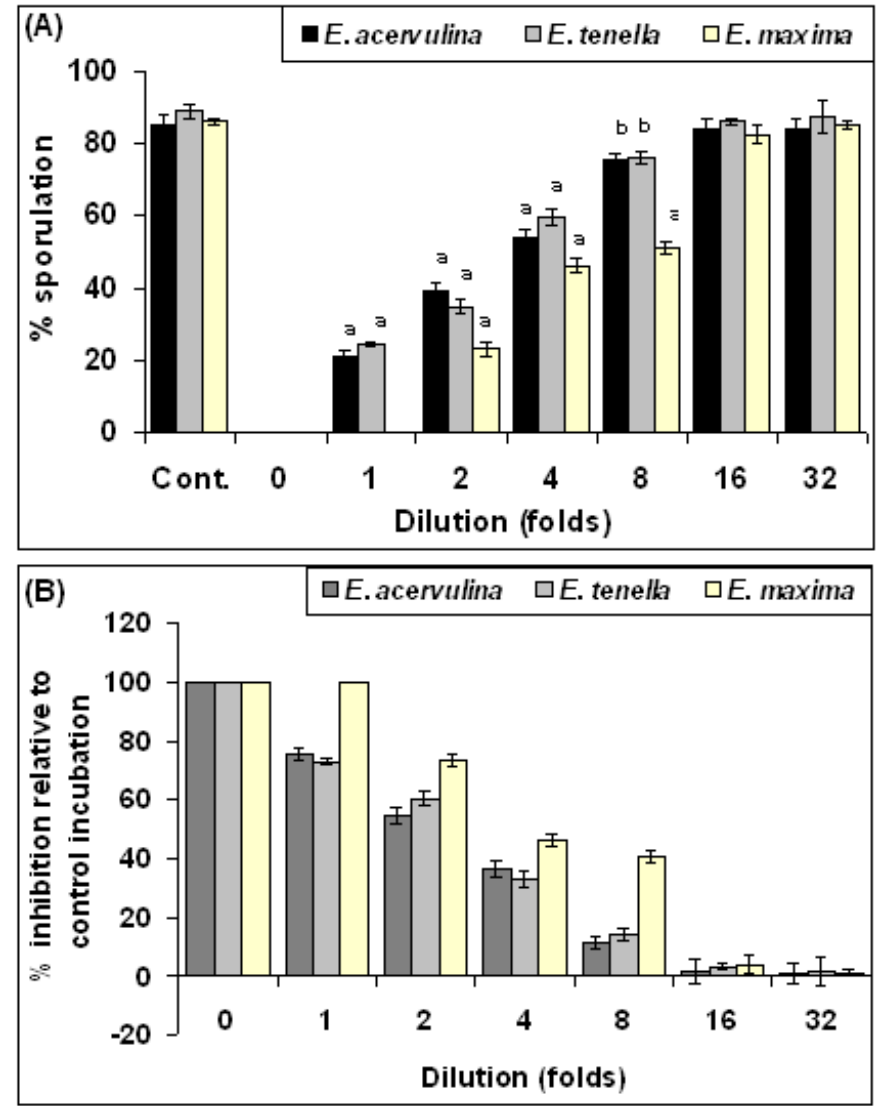

Figure 1. Percentage sporulation (A) and \% inhibition of sporulation (B) of Eimeria acervulina, E. tenella and E. maxima oocysts recovered from incubations containing water only (control) or Lactobacillus rhamnosus undiluted (0) and diluted (1-32 folds) cell-free supernatant. Results are the mean \pm S.E.M. of two independent experiments of triplicate incubations. Percentage inhibition of sporulation is expressed as a percentage of inhibition relative to sporulation in control incubations without cell-free supernatant. Significantly different from the value in the control incubation: $\mathrm{a}=\mathrm{P}<0.0001, \mathrm{~b}=\mathrm{P}<0.05$.

\subsection{Effect of L. rhamnosus cell-free supernatant on fecal oocyst output and sproulation of Eimeria tenella oocysts in chickens}

As shown in Fig. 2, chickens fed diets supplemented with 1\% and 3\% CFS shed significantly fewer oocysts than their counterpart's infected-non-treated birds ( $\mathrm{P}<0.0001)$. Chickens fed $1 \%$ and 3\% CFSsupplemented diets showed a reduction of $20 \%$ and $37 \%$, respectively in fecal oocyst output following E. tenella infection relative to the birds in the control group fed standard diet.

The influence of treatments on the viability of the oocysts as judged by the ability of oocysts, recovered from chickens fed unsupplemented diet (control group) and those fed CFS-supplemented diets, to sporulate under in vitro conditions is summarized in Fig. 3. About $91 \%$ of the oocysts recovered from the chickens fed the control diet were managed to sporulate (Fig. 3A). Supplementation of the diet with $1 \%$ or $3 \%$ of CFS led to a significant decrease in sporulation rate $(\mathrm{P}<0.0001)$ when compared to the oocysts shed by the control group (Fig. 3B). This corresponds to $23.9 \%$ and $49.3 \%$ reduction in sporulation of oocysts recovered from chickens fed the diets supplemented with $1 \%$ and $3 \%$ CFS, respectively relative to the control group fed standard diet.

Although there was no significant differences in body weight gains among the groups fed control diet or CFS-supplemented diets, weight gains of birds on the $1 \%$ and $3 \%$ CFS-supplemented diets were $4 \%$ and $7.8 \%$, respectively higher than those of birds fed control diet (data not shown). 


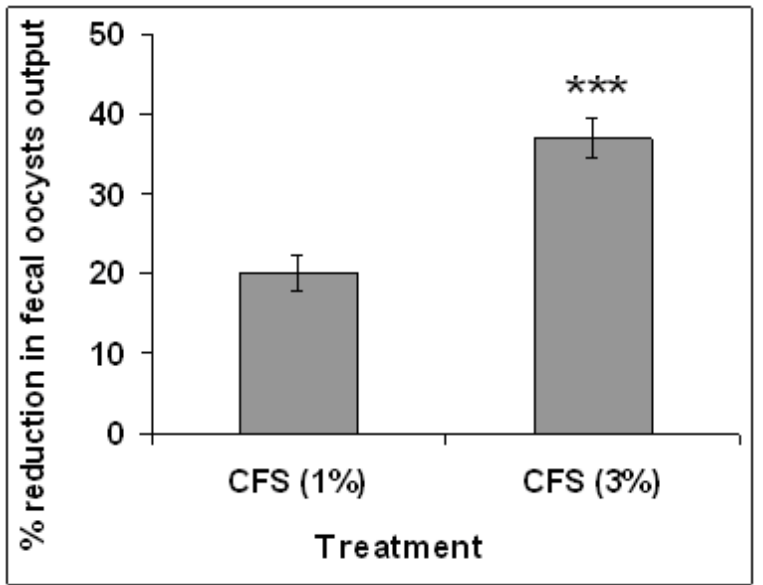

Figure 2. Effect of diet supplemented with Lactobacillus rhamnosus cell-free supernatant (CFS) on oocyst per gram of feces shed from chickens challenged with Eimeria tenella at 14 days of age. Percentage reduction in oocyst output is expressed as a percentage of reduction relative to chickens fed the control diet (unsupplemented with CFS). Results are the mean \pm S.E.M. $(n=9)$. Significantly different from CFS (1\%) group: *** $\mathrm{P}<0.0001$.
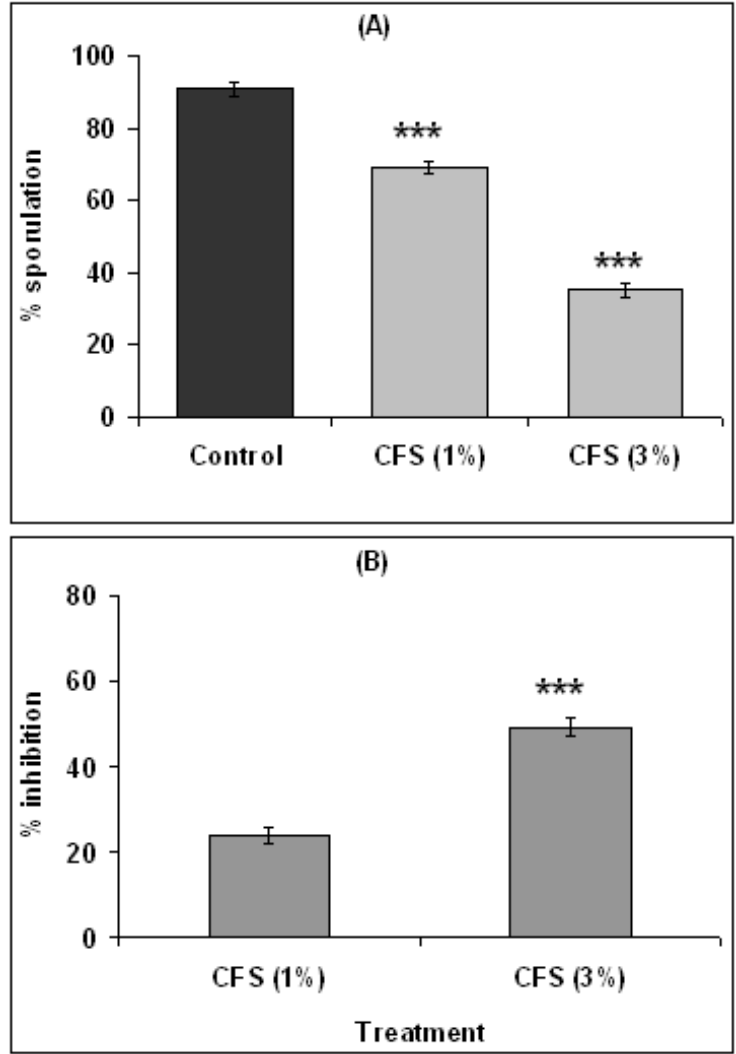

Figure 3. Percentage sporulation (A) and \% inhibition of sporulation (B) of Eimeria tenella oocysts recovered from chickens fed the control diet only (control group) or chickens fed diet supplemented with either $1 \%$ or $3 \%$ Lactobacillus rhamnosus cell-free supernatant (CFS). Results are the mean \pm S.E.M. (n=9). Percentage inhibition of sporulation is expressed as a percentage of inhibition relative to sporulation of oocysts recovered from chickens fed the control diet (unsupplemented with CFS). Significantly different from control group (A) or from CFS (1\%) group (B): *** $\mathrm{P}<0.0001$.

\section{Discussion}

This study shows for the first time that cell-free supernatants obtained from L. rhamnosus cultures significantly reduced the sporulation of three species of Eimeria, E. acervulina, E. tenella and E. maxima under laboratory conditions. Chickens fed $1 \%$ or $3 \%$ CFS- supplemented diets in E. tenella-infected groups shed significantly less oocysts than their counterparts fed control diet. More importantly, the sporulation rate of the 
oocysts recovered from the CFS-supplemented birds was significantly lower than that of the oocysts recovered from the infected birds fed the control diet. These results are of a major importance as contamination of poultry houses with coccidian oocysts is very difficult to control [14] and all attempts to eradicate infections with Eimeria spp. have failed [15]. Some studies have indicated that E. tenella oocysts, for example, can survive in soil for 9 months [16].

Although the number of oocysts shed by the infected birds is an important parameter, the most important factor in the epidemiology of coccidia is the ability of the oocysts to sporulate, which constitute the reservoir of infection. In fact, coccidian infection occurs only when chickens ingest sporulated oocysts and infected birds excrete oocysts with their feces, of which a number will sporulate. Subsequently, sporulated oocysts are ingested by the same or other chickens, hereby spreading the infection. Consequently, the significant reduction in sporulation of the fecal oocyst shed by birds fed CFS-supplemented diet will lead to less contamination with infective coccidian oocysts. Similarly, Tierney et al. [17] studied the effect of extracellular components of indigenous chicken Lactobacillus species on invasion of E. tenella under in vitro conditions and found that extracellular metabolic factors secreted by Lactobacillus species into the surrounding media were shown to inhibit parasite invasion and these factors appeared to be heat stable. The researchers concluded that the natural microflora of poultry can provide a source of E. tenella-inhibiting Lactobacillus species in vitro, and thus may contribute to the control of Eimeria infection. In addition, Dalloul et al. [18] reported that chickens on a Lactobacillus-supplemented feed had reduced oocyst output compared to controls after challenge with $E$. acervulina. Moreover, Foster et al. [11] showed that an incubation time as short as $24 \mathrm{~h}$ at $37{ }^{\circ} \mathrm{C}$ in $L$. acidophilus or L. rhmnosus supernatant reduced bovine C. parvum oocyst viability by up to $86 \%$. In vitro studies have also demonstrated Lactobacillus influence on Trichomonas vaginalis [19] and Giardia intestinalis [20]. Recently, Lee et al. [21] studied the effects of Pediococcus- and Saccharomyces-based probiotic $\left(\right.$ MitoMax $^{\circledR}$ ) on coccidiosis in broiler chickens and found that birds fed MitoMax ${ }^{\circledR}$-supplemented diets and infected with E. acervulina or E. tenella shed significantly less oocysts than control-infected birds. The researchers also found no significant difference in body weight gains among the groups fed regular or MitoMaxsupplemented diets.

In general, the reduction in sporulation of the oocysts of E. tenella observed in this study in combination with previous reported reduction in invasion of $E$. tenella under in vitro conditions [17] may support the hypothesis that Lactobacillus species produce antimicrobial compounds harmful to coccidian oocysts.

Considering the large number of different groups of chemical compounds present in LAB metabolites, it is most likely that the anticoccidial activity observed in the present study is not attributable to one specific mechanism but to synergistic effects targeting the oocysts. Possible components of LAB metabolites include short chain fatty acids such as lactate and acetate which lower the local $\mathrm{pH}$; bacteriocins; hydrophobic organic compounds; and strong oxidative agents such as hydrogen peroxides [22-24]. Accordingly, more studies are needed to identify the products found in the bacterial cell-free supernatants, their mode of action against coccidian oocyst and the viability of the sporulated oocysts derived from incubations containing different concentrations of CFS. This will be the objectives of further studies by our group.

\section{Conclusion}

In conclusion, the results show for the first time that CFS from L. rhamnosus cultures have anticoccidial activity as evidenced by their ability to decrease significantly the sporulation of the oocysts of three species of Eimeria, namely E. tenella E. acervulina and E. maxima under in vitro conditions. Moreover, bacterial CFS also showed anticoccidial effects against experimental infection with $E$. tenella as evidenced by their ability to reduce the numbers of oocysts shed and the sporualtion rate.

\section{Acknowledgements}

The author would like to thank Mr James Liu for the technical support.

\section{References}

[1]. Cox, F.E.G. (1998). Control of coccidiosis: lessons from other sporozoa. International Journal for Parasitology, 28: 165-179.

[2]. Allen, P.C. and Fetterer R.H. (2002). Recent advances in biology and immunobiology of Eimeria species and in diagnosis and control of infection with these coccidian parasites of poultry. Clinical Microbiolgy Reviews, 15: 58-65.

[3]. Chapman, H.D. (1998). Evaluation of the efficacy of anticoccidial drugs against Eimeria species in the fowl. International Journal for Parasitology, 28: 1141-1144.

[4]. Yadav, A. and Gupta, S.K. (2001). Study of resistance against some ionophores in Eimeria tenella field isolates, Veterinary Parasitology, 102: 69-75.

[5]. Lehto, E.M. and Salminen, S.J. (1997). Inhibition of Salmonella typhimurium adhesion to Caco-2 cell cultures by Lactobacillus strain GG spent culture supernatant: only a pH effect? FEMS Immunology and Medical Microbiology, 18: $125-132$.

[6]. Rolfe, R.D. (2000). The role of probiotic cultures in the control of gastrointestinal health. Journal of Nutrition, 130: 396S-402S

[7]. Belfiore, C., Castellana, P. and, Vignolo, G. (2007). Reduction of Escherichia coli population following treatment with bacteriocins from lactic acid bacteria and chelators. Food Microbiology, 24: 223-229. 
Bacteria versus parasites: Effect of Lactobacillus rhamnosus cell-free supernatant on the.....

[8]. Alak, J.I., Wolf, B.W., Mdurvwa, E.G., Pimentel-Sith, G.E. and Adeyemo, O.(1997). Effect of Lactobacillus reuteri on intestinal resistance to Cryptosporidium parvum infection in a murine model of acquired immunodeficiency syndrome. Journal of Infectious Diseases, 175: 218-221

[9]. Alak, J.I., Wolf, B.W., Mdurvwa, E.G., Pimentel-Smith, G.E., Kolavala, S., Abdelrahman, H. and Suppiramaniama, V. (1999). Supplementation with Lactobacillus reuteri or L. acidophilus reduced intestinal shedding of Cryptosporidium parvum oocysts in immunodeficient C57BL/6 mice. Cellular and Molecular Biology, 45: 855-863.

[10]. Rotkiewicz, T., Rotkiewicz, Z., Depta, A. and Kander, M. (2001). Effect of Lactobacillus acidophilus and Bifidobacterium sp. on the course of Cryptosporidium parvum invasion in new-born piglets. Bulletin of the Veterinary Institute in Pulawy, 45: 187-195.

[11]. Foster, J.C., Glass, M.D., Courteny, P.D., Ward, L.A., 2003. Effect of Lactobacillus and Bifidobacterium on Cryptosporidium parvum oocyst viability. Food Microbiology, 20: 351-357.

[12]. Molan, A.L., Liu, Z. and DE, S. (2009). Effect of pine bark (Pinus radiata) extracts on sporulation of coccidian oocysts. Folia Parasitologica, 56: 1-5.

[13]. Haug, A., Williams, R. B. and Larsen, S. (2006). Counting coccidial oocysts in chicken feces: a comparative study of a standard McMaster technique and a new rapid method. Veterinary Parasitology, 136: 233-242.

[14]. Ryley, J. F. (1980). Recent development in coccidian biology; where do we go from here? Parasitology, 80: 189-209.

[15]. Graat, E.A.M., Henken, A.M., Piloeger, H.W., Noordhuizen, J.T.M. and Vertommen, M.H. (1994). Rate and course of sporulation of oocysts of Eimeria acervulina under different environmental conditions. Parasitology, 108: 497-502.

[16]. Marion, M.F. and Wehr, E.E. (1949). Survival of Eimeria acervulina, E. tenella and E. maxima oocysts in soil under various field conditions. Annals of Tropical Medicine and Parasitology, 52: 468-472.

[17]. Tierney, J., Gowing, H., Van Sinderen, D., Flynn, S., Stanley, L., Mchardy, N., Hallahan, S. and Mulcahy, G. (2004). In vitro inhibition of Eimeria tenella invasion by indigenous chicken Lactobacillus species. Veterinary Parasitology, 122: 171-182.

[18]. Dalloul, R.A., Lillehoj, H.S., Sheliem, T.A. and Doerr, J.A. (2003). Enhanced mucosal immunity against Eimeria acervulina in broilers fed a Lactobacillus-based probiotic. Poultry Science, 82: 62-66.

[19]. McGrory, T., Meysick, K., Lemchuk-Favel, L.T. and Garber, G.E. (1994). The interaction of Lactobacillus acidophilus and Trichomonas vaginalis in vitro. Journal of Parasitology, 80: 50-54.

[20]. Perez, P.F., Minnaard, J., Rouvet, M., Knabenhans, C., Brassart, D., De Antoni, G.L. and Schiffrin, E.J. (2001). Inhibition of Giardia intestinalis by extracellular factors from lactobacilli: an in vitro study. Applied and Environmental Microbiology, 67: 5037-5042.

[21]. Lee, S., Lillehoj, H.S., Park, D.W., Hong,Y.H. and Lin, J.J. (2007). Effects of Pediococcus- and Saccharomyces-based probiotic $\left(\right.$ MitoMax $\left.^{\circledR}\right)$ on coccidiosis in broiler chickens. Comparative Immunology, Microbiology and Infectious Diseases, 30: 261-268.

[22]. Ouwehand, C. and Vesterlund, S. (2004). Antimicrobial components from lactic acid bacteria. In: S. Salminen, A. Von Wright and A. Ouwehand, Editors, Lactic Acid Bacteria - Microbiological and Functional Aspects, Marcel Dekker, Inc., New York, pp. 375395.

[23]. Reid, G. (1999). The scientific basis for probiotic strains of Lactobacillus. Applied and Environmental Microbiology, 65: 37633766.

[24]. Vaughan, E.E., Mollet, B. and Devos, W.M. (1999). Functionality of probiotics and intestinal lactobacilli: light in the intestinal tract tunnel. Current Opinion in Biotechnology, 10: 505-510. 